PROCEEDINGS OF THE

AMERICAN MATHEMATICAL SOCIETY

Volume 134, Number 1, Pages 215-228

S 0002-9939(05)08165-7

Article electronically published on June 29, 2005

\title{
TOPOLOGICAL ENTROPY AND AF SUBALGEBRAS OF GRAPH $C^{*}$-ALGEBRAS
}

\author{
JA A JEONG AND GI HYUN PARK
}

(Communicated by David R. Larson)

\begin{abstract}
Let $\mathcal{A}_{E}$ be the canonical AF subalgebra of a graph $C^{*}$-algebra $C^{*}(E)$ associated with a locally finite directed graph $E$. For Brown and Voiculescu's topological entropy $h t\left(\Phi_{E}\right)$ of the canonical completely positive map $\Phi_{E}$ on $C^{*}(E), h t\left(\Phi_{E}\right)=h t\left(\left.\Phi_{E}\right|_{\mathcal{A}_{E}}\right)=h_{l}(E)=h_{b}(E)$ is known to hold for a finite graph $E$, where $h_{l}(E)$ is the loop entropy of Gurevic and $h_{b}(E)$ is the block entropy of Salama. For an irreducible infinite graph $E$, the inequality $h_{l}(E) \leq h t\left(\Phi_{E} \mid \mathcal{A}_{E}\right)$ has recently been known. It is shown in this paper that$$
h t\left(\Phi_{E} \mid \mathcal{A}_{E}\right) \leq \max \left\{h_{b}(E), h_{b}\left({ }^{t} E\right)\right\},
$$

where ${ }^{t} E$ is the graph $E$ with the direction of the edges reversed. Some irreducible infinite graphs $E_{p}(p>1)$ with $h t\left(\left.\Phi_{E}\right|_{\mathcal{A}_{E_{p}}}\right)=\log p$ are also examined.

\section{INTRODUCTION}

Voiculescu 22 introduced a notion of topological entropy $h t(\alpha)$ for an automorphism $\alpha$ of a nuclear unital $C^{*}$-algebra $A$ to measure the growth of $\alpha^{n}$ as $n \rightarrow \infty$ using the fact that a nuclear $C^{*}$-algebra has the completely positive approximation property. The definition extends very well to automorphisms of exact $C^{*}$-algebras (as done by Brown in [4]) due to the deep result by Kirchberg [13] that exact $C^{*}$ algebras are nuclearly embeddable. But without effort one can define $h t(\Phi)$ even for a completely positive (cp) map on an exact $C^{*}$-algebra as described in [2. Since a $C^{*}$-subalgebra of an exact $C^{*}$-algebra is always exact, if $\Phi: A \rightarrow A$ is a cp map on an exact $C^{*}$-algebra $A$ and $B$ is a $\Phi$-invariant $C^{*}$-subalgebra of $A$, then $h t\left(\left.\Phi\right|_{B}\right)$ can be defined and the monotonicity $h t\left(\left.\Phi\right|_{B}\right) \leq h t(\Phi)$ holds.

The topological entropy has been computed in several cases; for example, the equality $h t(\alpha * \beta)=\max \{h t(\alpha), h t(\beta)\}$ for the reduced free product automorphism $\alpha * \beta$ was proved in [1, when the free product is with amalgamation over a finitedimensional $C^{*}$-algebra. Also Dykema [9] showed that $h t(\alpha)=0$ for certain classes of automorphisms $\alpha$ of reduced amalgamated free products of $C^{*}$-algebras, which turns out to extend Størmer's result [21 that the Connes-Størmer entropy of the free shift automorphism of the $\mathrm{II}_{1}$-factor $L\left(F_{\infty}\right)$ is zero.

In this paper we are concerned with the topological entropy of the shift type cp maps on $C^{*}$-algebras arising from directed graphs. A typical one is the canonical

Received by the editors March 22, 2004 and, in revised form, August 26, 2004.

2000 Mathematics Subject Classification. Primary 46L05, 46L55.

The first author was partially supported by KOSEF R14-2003-006-01000-0.

The second author was partially supported by KOSEF R01-2001-000-00001-0.

(C)2005 American Mathematical Society 
cp map $\Phi_{A}: \mathcal{O}_{A} \rightarrow \mathcal{O}_{A}$ of the Cuntz-Krieger algebra $\mathcal{O}_{A}$ given by

$$
\Phi_{A}(x)=\sum_{i=1}^{n} s_{i} x s_{i}^{*},
$$

where $s_{1}, \ldots, s_{n}$ are the partial isometries that generate $\mathcal{O}_{A}$. The reason we call $\Phi_{A}$ shift type is that $\mathcal{O}_{A}$ contains a $\Phi_{A}$-invariant commutative $C^{*}$-subalgebra $\mathcal{D}_{A}$ which is isomorphic to $C\left(X_{A}\right)$ in such a way that the restriction $\left.\Phi_{A}\right|_{\mathcal{D}_{A}}$ corresponds to the shift map $\sigma_{X_{A}}$ on the (compact) shift space $X_{A}$ associated with the incidence matrix $A$. The topological entropy of $\Phi_{A}$ is then computed (see [5, 2, 11, 19]) as $h t\left(\Phi_{A}\right)=\log r(A)(r(A)$ is the spectral radius of $A)$. But $\log r(A)=h_{\text {top }}\left(X_{A}\right)$ is a well-known fact, so that one can deduce by [8] that $h t\left(\Phi_{A}\right)=h t\left(\Phi_{A} \mid \mathcal{D}_{A}\right)$. On the other hand, $\mathcal{O}_{A}$ also contains another important $\Phi_{A}$-invariant $C^{*}$-subalgebra $\mathcal{A}_{A}$ which is an AF algebra with $\mathcal{D}_{A} \subset \mathcal{A}_{A}$. Thus by monotonicity of entropy, we have $h t\left(\Phi_{A}\right)=h t\left(\left.\Phi_{A}\right|_{\mathcal{A}_{A}}\right)=h t\left(\left.\Phi_{A}\right|_{\mathcal{D}_{A}}\right)$.

The Cuntz-Krieger algebras $\mathcal{O}_{A}$ are now well understood as graph $C^{*}$-algebras $C^{*}(E)=C^{*}\left(s_{e}, p_{v}\right)$ associated with finite directed graphs $E$, and the cp map $\Phi_{A}$ of $\mathcal{O}_{A}$ is interpreted as the map $\Phi_{E}: C^{*}(E) \rightarrow C^{*}(E)$ given by $\Phi_{E}(x)=\sum_{e \in E^{1}} s_{e} x s_{e}^{*}$. Hence if $E$ is a finite graph (possibly with sinks) which contains an infinite path, it follows that $h t\left(\Phi_{E}\right)=h t\left(\left.\Phi_{E}\right|_{\mathcal{A}_{E}}\right)=h t\left(\left.\Phi_{E}\right|_{\mathcal{D}_{E}}\right)=\log r\left(A_{E}\right)$, where $\mathcal{A}_{E}$ is the AF subalgebra of $C^{*}(E)$ corresponding to $\mathcal{A}_{A}$ in $\mathcal{O}_{A}$ and $A_{E}$ is the edge matrix of $E$ (see [1]).

If $E$ is infinite but locally finite, then the map $\Phi_{E}$ is known to be a contractive cp map, and furthermore if $E$ is irreducible and $\mathcal{A}_{E}$ is the canonical AF subalgebra of $C^{*}(E)$, the inequality $h_{l}(E) \leq h t\left(\left.\Phi_{E}\right|_{\mathcal{A}_{E}}\right)$ is known to hold [11]. The purpose of the present paper is then to give an upper bound for $h t\left(\left.\Phi_{E}\right|_{\mathcal{A}_{E}}\right)$, and we actually prove the following (see Theorem 3.10):

$$
h t\left(\left.\Phi_{E}\right|_{\mathcal{A}_{E}}\right) \leq \max \left\{h_{b}(E), h_{b}\left({ }^{t} E\right)\right\} .
$$

In particular, for an irreducible infinite graph $E_{p}$ constructed in [20] so that $h_{l}\left(E_{p}\right)$ $=h_{b}\left(E_{p}\right)=\log p(p>1)$, we have $h t\left(\left.\Phi_{E_{p}}\right|_{\mathcal{A}_{E_{p}}}\right)=\log p$.

We believe that the result would be helpful to compute the entropy $h t\left(\Phi_{E}\right)$ of $\Phi_{E}$ on the whole graph $C^{*}$-algebra $C^{*}(E)$.

\section{Preliminaries}

2.1. Graph $C^{*}$-algebras. A (directed) graph is a quadruple $E=\left(E^{0}, E^{1}, r, s\right)$ of the vertex set $E^{0}$, the edge set $E^{1}$, and the range, source maps $r, s: E^{1} \rightarrow E^{0}$. A family $\left\{p_{v}, s_{e} \mid v \in E^{0}, e \in E^{1}\right\}$ of mutually orthogonal projections $p_{v}$ and partial isometries $s_{e}$ is called a Cuntz-Krieger E-family if the following relations hold:

$$
\begin{aligned}
& s_{e}^{*} s_{e}=p_{r(e)}, \quad s_{e} s_{e}^{*} \leq p_{s(e)}, \\
& p_{v}=\sum_{s(e)=v} s_{e} s_{e}^{*}, \quad \text { if } 0<\left|s^{-1}(v)\right|<\infty .
\end{aligned}
$$

The graph $C^{*}$-algebra $C^{*}(E)$ is then defined to be a $C^{*}$-algebra generated by a universal Cuntz-Krieger $E$-family (see [16, 17, 3]). If $E$ is row-finite, that is, each vertex emits only finitely many vertices, the relations can be written as (with the 
edge matrix $A_{E}$ of $E$ )

$$
s_{e}^{*} s_{e}=\sum_{f \in E^{1}} A_{E}(e, f) s_{f} s_{f}^{*} ;
$$

hence the family is also called a Cuntz-Krieger $A_{E}$-family.

Given a $\{0,1\}$ matrix $B$ such that each row has only finitely many non-zero entries (row-finite), let $E$ be the graph with the vertex matrix $B$. Then by definition $C^{*}(E)$ is generated by a Cuntz-Krieger $A_{E}$-family. But it is also generated by a Cuntz-Krieger $B$-family [17, Proposition 4.1]. Hence many results on $C^{*}$-algebras of $\{0,1\}$ matrices can be applied to graph $C^{*}$-algebras even though not all $\{0,1\}$ matrices can occur as edge matrices of some graphs.

We call a graph $E$ locally finite if each vertex receives and emits only finitely many edges. Throughout this paper we consider only locally finite graphs and adopt the notation in [16. If a finite path $\alpha \in E^{*}$ of length $|\alpha|>0$ is a return path, that is, $s(\alpha)=r(\alpha)$, then $\alpha$ is called a loop at $v=s(\alpha)$. A graph $E$ is said to be irreducible if for any two vertices $v, w$ there is a finite path $\alpha \in E^{*}$ with $s(\alpha)=v$ and $r(\alpha)=w$. It is known that if $E$ is irreducible and every loop has an exit, then $C^{*}(E)$ is simple $([16])$.

2.2. Topological entropy of cp maps. Let $A$ be a $C^{*}$-algebra, $\pi: A \rightarrow B(H)$ a faithful *-representation, and $\operatorname{Pf}(A)$ the set of all finite subsets of $A$. For $\omega \in$ $\operatorname{Pf}(A)$ and $\delta>0$, put

$$
\begin{gathered}
C P A(\pi, A)=\{(\phi, \psi, B) \mid \phi: A \rightarrow B, \psi: B \rightarrow B(H) \text { are contractive cp maps } \\
\text { and } \left.B \text { is a } C^{*} \text {-algebra with } \operatorname{dim} B<\infty\right\}, \\
\operatorname{rcp}(\pi, \omega, \delta)=\inf \{\operatorname{rank}(B) \mid(\phi, \psi, B) \in C P A(\pi, A),\|\psi \circ \phi(x)-\pi(x)\|<\delta, \\
\text { for all } x \in \omega\},
\end{gathered}
$$

where $\operatorname{rank}(B)$ denotes the dimension of a maximal abelian subalgebra of $B$.

Since the cp $\delta$-rank $\operatorname{rcp}(\pi, \omega, \delta)$ is independent of the choice of $\pi(2,4])$ and graph $C^{*}$-algebras $C^{*}(E)$ are nuclear $([15)$ we may write $\operatorname{rcp}(\omega, \delta)$ for $\operatorname{rcp}(\pi, \omega, \delta)$ assuming that $C^{*}(E) \subset B(H)$ for a Hilbert space $H$.

Definition $2.1([2,4,22])$. Let $A \subset B(H)$ be a $C^{*}$-algebra and let $\Phi: A \rightarrow A$ be a cp map. Put

$$
\begin{aligned}
h t(\Phi, \omega, \delta) & =\limsup _{n \rightarrow \infty} \frac{1}{n} \log \left(\operatorname{rcp}\left(\bigcup_{i=0}^{n-1} \Phi^{i}(\omega), \delta\right)\right), \\
h t(\Phi, \omega) & =\sup _{\delta>0} h t(\Phi, \omega, \delta) .
\end{aligned}
$$

Then $h t(\Phi):=\sup _{\omega \in P f(A)} h t(\Phi, \omega)$ is called the topological entropy of $\Phi$.

Remark 2.2. We refer the reader to [2] and [4] for the following useful properties and their proofs. Let $A$ be an exact $C^{*}$-algebra and let $\Phi: A \rightarrow A$ be a cp map.

(a) If $\theta: A \rightarrow B$ is a $C^{*}$-isomorphism, then $h t(\Phi)=h t\left(\theta \Phi \theta^{-1}\right)$.

(b) Let $\tilde{A}$ be the unital $C^{*}$-algebra obtained by adjoining a unit and let $\tilde{\Phi}$ : $\tilde{A} \rightarrow \tilde{A}$ be the extension of $\Phi$. Then $h t(\tilde{\Phi})=h t(\Phi)$.

(c) If $A_{0} \subset A$ is a $\Phi$-invariant $C^{*}$-subalgebra of $A, h t\left(\left.\Phi\right|_{A_{0}}\right) \leq h t(\Phi)$. 
We will use the following Arveson's extension theorem several times.

Arveson Extension Theorem (see [4). Let $A$ be a unital $C^{*}$-algebra, $S \subset A$ a unital subspace with $S=S^{*}$, and $\phi: S \rightarrow B$ a contractive cp map where $B=B(H)$ or $\operatorname{dim}(B)<\infty$. Then $\phi$ extends to a cp map $\bar{\phi}: A \rightarrow B$. If $S$ is a $C^{*}$-subalgebra of $A$, then we obtain a unital cp extension of $\phi$ even when $S$ does not contain the unit of $A$.

If $E$ is a locally finite graph, the map $\Phi_{E}: C^{*}(E) \rightarrow C^{*}(E)$, defined by

$$
\Phi_{E}(x)=\sum_{e \in E^{1}} s_{e} x s_{e}^{*},
$$

is well defined, contractive and completely positive [11. For a finite graph $E$, the topological entropy $h t\left(\Phi_{E}\right)$ has been obtained as follows (see [2], [5], 19, or [11).

Theorem 2.3. Let $E$ be a finite graph possibly with sinks and let $A_{E}$ be the edge matrix of $E$. If $E$ contains an infinite path, then

$$
h t\left(\Phi_{E}\right)=\log r\left(A_{E}\right),
$$

where $r\left(A_{E}\right)$ is the spectral radius of $A_{E}$.

By $h_{t o p}(X)$ we denote the topological entropy of a compact space $(X, T)$ together with a continuous map $T: X \rightarrow X$ (for a definition, see [23, Chapter 7]). Let $E$ be a locally finite infinite graph with no sinks and $X_{E}$ the locally compact shift space of (one-sided) infinite paths with the one point compactification $\bar{X}_{E}$. The first identity in the following theorem is shown for the doubly infinite path space of $E$ by Gurevic [10. See Remark 3.6(a) for a definition of the entropy $h\left(X_{E}\right)$ for a finite graph $E$.

Theorem 2.4 ([11, Theorem 4.4]). Let $E$ be a locally finite irreducible infinite graph. Then

$$
h_{t o p}\left(\bar{X}_{E}\right)=\sup _{E^{\prime}} h\left(X_{E^{\prime}}\right) \leq h t\left(\Phi_{E}\right),
$$

where the supremum is taken over all the finite (irreducible) subgraphs of E.

\section{MAIN RESULTS}

Throughout this section $E$ will denote a locally finite infinite graph unless stated otherwise. For a path $\alpha \in E^{*}$, let $\alpha^{0}$ be the set of vertices lying on $\alpha=\alpha_{1} \cdots \alpha_{n}$, that is, $\alpha^{0}=\left\{s\left(\alpha_{1}\right), r\left(\alpha_{1}\right), \ldots, r\left(\alpha_{n}\right)\right\}$. For a fixed vertex $v$ we consider the following subsets of finite paths $E^{n}$ of length $n$ :

(i) $E^{n}(v)=\left\{\alpha \in E^{n} \mid v \in \alpha^{0}\right\}$,

(ii) $E_{s}^{n}(v)=\left\{\alpha \in E^{n} \mid s(\alpha)=v\right\}$,

(iii) $E_{s}^{n}(v)^{\star}=\left\{\alpha \in E_{s}^{n}(v) \mid r\left(\alpha_{i}\right) \neq v, 1 \leq i \leq n\right\}$,

(iv) $E_{l}^{n}(v)=\left\{\alpha \in E^{n} \mid \alpha\right.$ is a loop at $\left.v\right\}$.

Similarly we can think of $E_{r}^{n}(v)$ and $E_{r}^{n}(v)^{\star}$.

Definition 3.1. Let $E$ be a graph and $v \in E^{0}$. Put

$$
h_{l}(E, v)=\underset{n}{\limsup } \frac{1}{n} \log \left|E_{l}^{n}(v)\right| \text { and } h_{b}(E, v)=\limsup _{n} \frac{1}{n} \log \left|E_{s}^{n}(v)\right| .
$$

The loop entropy $h_{l}(E)$ and the block entropy $h_{b}(E)$ of $E$ are defined by

$$
h_{l}(E):=\sup _{v \in E^{0}} h_{l}(E, v) \text { and } h_{b}(E):=\sup _{v \in E^{0}} h_{b}(E, v) .
$$


If $E$ is irreducible, $h_{l}(E, v)$ and $h_{b}(E, v)$ are independent of the choice of a vertex $v$ [20; hence $h_{l}(E)=h_{l}(E, v)$ and $h_{b}(E)=h_{b}(E, v)$ for any $v \in E^{0}$. Let ${ }^{t} E$ denote the graph $E$ with the direction of all edges reversed. Then $h_{l}(E)=h_{l}\left({ }^{t} E\right)$ is immediate while $h_{b}(E) \neq h_{b}\left({ }^{t} E\right)$ in general as we will see in Example 3.3.

We will use the following notation for the infinite series with coefficients from (i)-(iv) above:

(i) $E(v, z):=\sum\left|E^{n}(v)\right| z^{n}$,

(ii) $E_{s}(v, z):=\sum\left|E_{s}^{n}(v)\right| z^{n}$,

(iii) ${ }^{\prime} E_{s}^{\star}(v, z):=\sum\left|E_{s}^{n}(v)^{\star}\right| z^{n}$,

(iv) $E_{l}(v, z):=\sum\left|E_{l}^{n}(v)\right| z^{n}$.

We denote the radius of convergence of the series $E_{s}^{\star}(v, z)$ by $R_{E_{s}^{\star}}$. Thus

$$
R_{E_{s}^{\star}}^{-1}=\limsup _{n \rightarrow \infty}\left|E_{s}^{n}(v)^{\star}\right|^{1 / n}
$$

Similarly $R_{E_{r}^{\star}}$ denotes the radius of convergence of $E_{r}^{\star}(v, z):=\sum\left|E_{r}^{n}(v)\right|^{\star} z^{n}$. As in [20, p.331], if $C_{v}^{(n)}$ is the number of sequences $v v_{i_{1}} \cdots v_{i_{n-1}}$ of vertices such that $v_{j} \neq v$ for $j=i_{1}, \ldots, i_{n-1},\left|C_{v}^{(n)}\right|=\left|E_{s}^{n-1}(v)^{\star}\right|$ and so the radius of convergence of $E_{s}^{\star}(v, z)$ coincides with that (denoted by $Q_{0}$ in 20$]$ ) of $\sum_{n} C_{v}^{(n)} z^{n}$. The following is Lemma (3.1) of [20].

Proposition $3.2([20)$. If $E$ is an irreducible graph, then

$$
h_{b}(E)=\max \left\{\log \left(R_{E_{s}^{\star}}^{-1}\right), h_{l}(E)\right\} .
$$

Note that if $E$ is irreducible, then $h_{b}\left({ }^{t} E\right)=\lim \sup \frac{1}{n} \log \left|E_{r}^{n}(v)\right|$ and so from the above proposition we have

$$
h_{b}\left({ }^{t} E\right)=\max \left\{\log \left(R_{E_{r}^{\star}}^{-1}\right), h_{l}(E)\right\} .
$$

The following example shows that $h_{b}(E) \neq h_{b}\left({ }^{t} E\right)$ in general.

Example 3.3. For each pair of positive real numbers $1<p \leq q$, Salama 20] constructed an irreducible infinite graph $E_{p, q}$ with

$$
h_{l}\left(E_{p, q}\right)=\log p \text { and } h_{b}\left(E_{p, q}\right)=\log q .
$$

As mentioned in the proof of [20, Theorem (3.9)], $E_{p, q}$ may be constructed to be a (uniformly) locally finite graph using the idea in [20, Example (3.7)].

For example, the following graph $E:=E_{2,8}$ satisfies $h_{l}(E)=\log 2$ and $h_{b}(E)=$ $\log 8$. There are 8 edges from the vertex $n$ to the vertex $n+1$ for each $n \geq 0$.

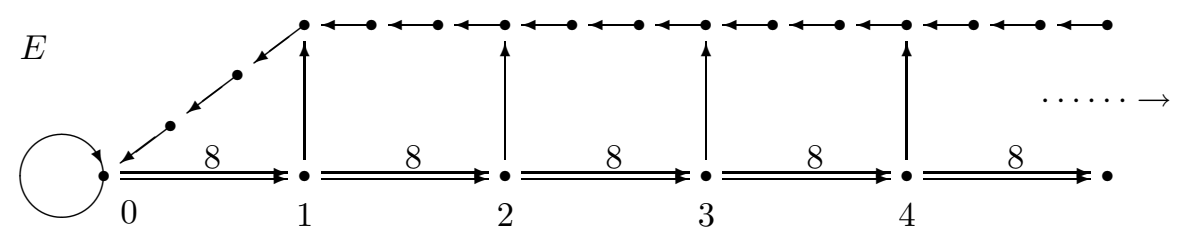

Now we first show that

$$
\log \left(R_{E_{r}^{\star}}^{-1}\right)<h_{l}(E)
$$


For a fixed vertex 0 we have

$$
\begin{aligned}
R_{E_{r}^{\star}}^{-1} & =\limsup _{n \rightarrow \infty}\left|E_{r}^{n}(0)^{\star}\right|^{1 / n} \\
& =\limsup _{n \rightarrow \infty} \mid\left.\left\{\alpha \in E_{r}^{n}(0) \mid s\left(\alpha_{i}\right) \neq 0, \text { for } 1 \leq i \leq n\right\}\right|^{1 / n} .
\end{aligned}
$$

Since

$$
\left|E_{r}^{4 k}(0)^{\star}\right|=1+8^{k-1}+8^{k-4}+8^{k-7}+\cdots,
$$

it follows that

$$
\limsup _{k \rightarrow \infty}\left|E_{r}^{4 k}(0)^{\star}\right|^{\frac{1}{4 k}}=8^{1 / 4} .
$$

But it is not hard to see that

$$
\limsup _{n \rightarrow \infty}\left|E_{r}^{n}(0)^{\star}\right|^{\frac{1}{n}}=\limsup _{k \rightarrow \infty}\left|E_{r}^{4 k}(0)^{\star}\right|^{\frac{1}{4 k}} .
$$

Hence $\log \left(R_{E_{r}^{\star}}^{-1}\right)=\log 8^{1 / 4}<\log 2=h_{l}(E)$. By $(1), h_{b}\left({ }^{t} E\right)=h_{l}(E)=\log 2$ and so we conclude that $h_{b}\left({ }^{t} E\right)<h_{b}(E)$.

Lemma 3.4. If $E$ is an irreducible graph, then the value

$$
\limsup _{n \rightarrow \infty} \frac{1}{n} \log \left|E^{n}(v)\right|
$$

is independent of the choice of a vertex $v$.

Proof. Let $v, w$ be two vertices of $E$. Then there exist two paths $\mu \in E^{k}, \nu \in E^{m}$ with $s(\mu)=r(\nu)=v, s(\nu)=r(\mu)=w$ because $E$ is irreducible. We assume that $\mu$ and $\nu$ have the smallest length, respectively. If $\alpha=\alpha_{1} \alpha_{2} \cdots \alpha_{n} \in E^{n}(v)$, then with $i_{0}=\min \left\{i \mid s\left(\alpha_{i}\right)=v\right\}$ write $\alpha=\alpha^{\prime} \alpha^{\prime \prime}$, where $\alpha^{\prime}=\alpha_{1} \cdots \alpha_{i_{0}-1}$ and $\alpha^{\prime \prime}=\alpha_{i_{0}} \cdots \alpha_{n}$ (if $\left.i_{0}=1, \alpha=\alpha^{\prime \prime}\right)$. Then the map

$$
E^{n}(v) \rightarrow E^{n+k+m}(w), \quad \alpha=\alpha^{\prime} \alpha^{\prime \prime} \mapsto \alpha^{\prime} \mu \nu \alpha^{\prime \prime}
$$

is injective; hence $\left|E^{n}(v)\right| \leq\left|E^{n+k+m}(w)\right|$ for each $n$. Therefore

$$
\begin{aligned}
\limsup _{n \rightarrow \infty} \frac{1}{n} \log \left|E^{n}(v)\right| & \leq \limsup _{n \rightarrow \infty} \frac{1}{n} \log \left|E^{n+k+m}(w)\right| \\
& \leq \limsup _{n \rightarrow \infty} \frac{1}{n} \log \left|E^{n}(w)\right| .
\end{aligned}
$$

Proposition 3.5. Let $E$ be an irreducible graph and $v_{0} \in E^{0}$.

(a) If $E$ is finite, then

$$
\limsup _{n \rightarrow \infty} \frac{1}{n} \log \left|E_{l}^{n}\left(v_{0}\right)\right|=\limsup _{n \rightarrow \infty} \frac{1}{n} \log \left|E^{n}\right| .
$$

In particular, $h_{l}(E)=h_{b}(E)=h_{b}\left({ }^{t} E\right)$.

(b) If $E$ is infinite, then

$$
\limsup _{n \rightarrow \infty} \frac{1}{n} \log \left|E^{n}\left(v_{0}\right)\right|=\max \left\{h_{b}(E), h_{b}\left({ }^{t} E\right)\right\} .
$$


Proof. (a) Let $E^{0}=\left\{v_{0}, v_{1}, \ldots, v_{k-1}\right\}$. Since $E$ is irreducible there exist finite paths $\left\{\mu_{i}, \nu_{i} \mid 0 \leq i \leq k-1\right\}$ such that $s\left(\mu_{i}\right)=r\left(\nu_{i}\right)=v_{0}, r\left(\mu_{i}\right)=v_{i}=s\left(\nu_{i}\right)$. Suppose $\left|\mu_{i}\right|=m_{i},\left|\nu_{j}\right|=l_{j}$. If $\alpha \in E^{n}$ is a path with $s(\alpha)=v_{i}, r(\alpha)=v_{j}$ then $\mu_{i} \alpha \nu_{j} \in E_{l}^{n+m_{i}+l_{j}}\left(v_{0}\right)$ is a loop at $v_{0}$. The map $\alpha \mapsto \mu_{i} \alpha \nu_{j}$ is not necessarily injective, but there exist at most $k_{0}$ paths in $E^{n}$ that have the same image in $E_{l}^{n+m_{i}+l_{j}}\left(v_{0}\right)$ under the map, where $k_{0}=\max _{i, j}\left\{m_{i}+l_{j}\right\}$. Hence we have

$$
\left|E^{n}\right| \leq k_{0} \cdot\left|\bigcup_{0 \leq i, j \leq k-1} E_{l}^{n+m_{i}+l_{j}}\left(v_{0}\right)\right| \leq k_{0} k^{2} \max _{i, j}\left|E_{l}^{n+m_{i}+l_{j}}\left(v_{0}\right)\right| .
$$

On the other hand, for each $n$, there exists a $k_{n} \in\left\{0, \ldots, k_{0}\right\}$ such that

$$
\left|E_{l}^{n+k_{n}}\left(v_{0}\right)\right|=\max _{i, j}\left|E_{l}^{n+m_{i}+l_{j}}\left(v_{0}\right)\right| .
$$

Then $\left|E^{n}\right| \leq k_{0} k^{2}\left|E_{l}^{n+k_{n}}\left(v_{0}\right)\right|$ and it follows that

$$
\limsup _{n \rightarrow \infty} \frac{1}{n} \log \left|E^{n}\right| \leq \limsup _{n \rightarrow \infty} \frac{1}{n} \log \left|E_{l}^{n}\left(v_{0}\right)\right| .
$$

(b) Note first that

$$
\begin{aligned}
\left|E^{n}\left(v_{0}\right)\right| & =\left|\bigcup_{k=0}^{n}\left\{\alpha \beta \mid \alpha \in E_{r}^{k}\left(v_{0}\right)^{\star}, \beta \in E_{s}^{n-k}\left(v_{0}\right)\right\}\right| \\
& =\sum_{k=0}^{n}\left|E_{r}^{k}\left(v_{0}\right)^{\star}\right|\left|E_{s}^{n-k}\left(v_{0}\right)\right|=\sum_{k=0}^{n}\left|\left({ }^{t} E\right)_{s}^{k}\left(v_{0}\right)^{\star}\right|\left|E_{s}^{n-k}\left(v_{0}\right)\right| .
\end{aligned}
$$

Then

$$
\begin{aligned}
E\left(v_{0}, z\right) & =\sum_{n}\left(\sum_{k=0}^{n}\left|\left({ }^{t} E\right)_{s}^{k}\left(v_{0}\right)^{\star}\right|\left|E_{s}^{n-k}\left(v_{0}\right)\right|\right) z^{n} \\
& =\left(\sum_{n}\left|\left({ }^{t} E\right)_{s}^{n}\left(v_{0}\right)^{\star}\right| z^{n}\right)\left(\sum_{n}\left|E_{s}^{n}\left(v_{0}\right)\right| z^{n}\right) \\
& =\left({ }^{t} E\right)_{s}^{\star}\left(v_{0}, z\right) \cdot E_{s}\left(v_{0}, z\right),
\end{aligned}
$$

so that the radius of convergence $R_{E}$ of $E\left(v_{0}, z\right)$ is equal to $\min \left\{R_{\left({ }^{t} E\right)_{s}^{\star}}, R_{E_{s}}\right\}$. Thus

$$
R_{E}^{-1}=\max \left\{R_{\left({ }^{t} E\right)_{s}^{\star}}^{-1}, R_{E_{s}}^{-1}\right\} .
$$

But Proposition 3.2 gives

$$
\log \left(R_{\left({ }^{t} E\right)_{s}^{\star}}^{-1}\right) \leq h_{b}\left({ }^{t} E\right),
$$

and also by definition $\log \left(R_{E_{s}}^{-1}\right)=h_{b}(E)$. Therefore

$$
\limsup _{n \rightarrow \infty} \frac{1}{n} \log \left|E^{n}\left(v_{0}\right)\right|=\log \left(R_{E}^{-1}\right) \leq \max \left\{h_{b}\left({ }^{t} E\right), h_{b}(E)\right\} .
$$


Remark 3.6. Let $E$ be a finite graph with the irreducible components $\left\{E_{i}\right\}_{i=1}^{s}$ so that the Perron eigenvalue of the edge matrix $A_{E}$ of $E$ is $\lambda_{E}=\max _{1 \leq i \leq s}\left\{\lambda_{E_{i}}\right\}$, where $\lambda_{E_{i}}$ is the Perron eigenvalue of the edge matrix of the irreducible graph $E_{i}$ (see [18, 4.4]).

(a) Assuming $\lambda_{E}=\lambda_{E_{1}}$ without loss of generality, we have from [18, Theorem 4.4.4] that

$$
h\left(X_{E}\right)=\log \lambda_{E}=\log \lambda_{E_{1}},
$$

where $h\left(X_{E}\right)=\lim _{n} \frac{1}{n} \log \left|E^{n}\right|$ is the topological entropy of $X_{E}$ (or $\Sigma_{E}$, the two-sided edge shift space). See [18, Definition 4.1.1] or [14, p.23] for the definition of $h\left(X_{E}\right)$. Since $\log \lambda_{E_{1}}=h\left(X_{E_{1}}\right)$ ([18, Theorem 4.3.1]) and $h\left(X_{E_{1}}\right)=h_{l}\left(E_{1}\right)=h_{b}\left(E_{1}\right)$ by Proposition 3.5(a) ( $E_{1}$ is irreducible), we see that all the entropies $h_{l}(E), h_{b}(E)$ and $h\left(X_{E}\right)$ are the same and equal to $\log \lambda_{E}$ because $\log \lambda_{E}=\log \lambda_{E_{1}}=h_{l}\left(E_{1}\right) \leq h_{l}(E) \leq h_{b}(E) \leq h\left(X_{E}\right)=$ $\log \lambda_{E}$.

(b) Since the eigenvalues of $A_{E}$ are exactly the eigenvalues of the $A_{E_{i}}$, by [18, Lemma 4.4.3] it follows that $\log \lambda_{E}=\log r\left(A_{E}\right)$. Thus by Theorem 2.3, $h t\left(\Phi_{E}\right)=h_{l}(E)=h_{b}(E)=h\left(X_{E}\right)$ for any finite graph $E$ which contains an infinite path (or a loop). If $E$ has no infinite paths, $h\left(X_{E}\right)=-\infty$ while $h t\left(\Phi_{E}\right) \geq 0$.

Let $E$ be an irreducible infinite graph and let $\mathcal{D}_{E}$ be the commutative $C^{*}$ subalgebra of $C^{*}(E)$ generated by the projections $\left\{p_{\alpha}=s_{\alpha} s_{\alpha}^{*} \mid \alpha \in E^{*}\right\}$. Then $\mathcal{D}_{E}=\overline{\operatorname{span}}\left\{p_{\alpha} \mid \alpha \in E^{*}\right\}$ and the map

$$
w: \mathcal{D}_{E} \rightarrow C_{0}\left(X_{E}\right), w\left(p_{\alpha}\right)=\chi_{[\alpha]},
$$

is a $C^{*}$-isomorphism such that $w\left(\left.\Phi_{E}\right|_{\mathcal{D}_{E}}\right) w^{-1}=\sigma_{E}^{*}$ [11. Here $\chi_{[\alpha]}$ is the characteristic function on the cylinder set $[\alpha]=\left\{\beta \in X_{E} \mid \beta=\alpha \beta^{\prime}\right\}$ which is both open and closed, and $\sigma_{E}^{*}: C_{0}\left(X_{E}\right) \rightarrow C_{0}\left(X_{E}\right)$ is the $*$-homomorphism induced by the shift map $\sigma_{E}$ on $X_{E}$, that is, $\sigma_{E}^{*}(f)=f \circ \sigma_{E}$ for $f \in C_{0}\left(X_{E}\right)$. By Remark $2.2(\mathrm{a}), h t\left(\left.\Phi_{E}\right|_{\mathcal{D}_{E}}\right)=h t\left(\sigma_{E}^{*}\right)$. But $h t\left(\sigma_{E}^{*}\right)=h t\left(\widetilde{\sigma_{E}^{*}}\right)$ by Remark $2.2(\mathrm{~b})$ and $h t\left(\widetilde{\sigma_{E}^{*}}\right)=$ $h_{\text {top }}\left(\bar{X}_{E}\right)$ by [8, Proposition 1.2]. On the other hand, $h_{t o p}\left(\bar{X}_{E}\right)=\sup _{E^{\prime} \subset E} h\left(X_{E^{\prime}}\right)$ is proved in [10, where the supremum is taken over all the finite subgraphs $E^{\prime}$ of $E$ (or equivalently, over all the irreducible finite subgraphs). If $\sup _{E^{\prime} \subset E} h\left(X_{E^{\prime}}\right)<\infty$, $h_{l}(E)=\sup _{E^{\prime} \subset E} h\left(X_{E^{\prime}}\right)$ is known (see [18, p.465]). If $\sup _{E^{\prime} \subset E} h\left(X_{E^{\prime}}\right)=\infty$, clearly $h_{l}(E)=\infty$ since $h\left(X_{E^{\prime}}\right)=h_{l}\left(E^{\prime}\right)$ for any finite graph $E^{\prime}$ (Remark 3.6(a)) and $h_{l}\left(E^{\prime}\right) \leq h_{l}(E)$. Thus $h_{t o p}\left(\bar{X}_{E}\right)=\sup _{E^{\prime} \subset E} h\left(X_{E^{\prime}}\right)=h_{l}(E)$ always holds for a locally finite irreducible infinite graph $E$. Hence we have

$$
h t\left(\left.\Phi_{E}\right|_{\mathcal{D}_{E}}\right)=h_{l}(E) .
$$

Put

$$
\mathcal{A}_{E}:=\overline{\operatorname{span}}\left\{s_{\alpha} s_{\beta}^{*}\left|\alpha, \beta \in E^{*},\right| \alpha|=| \beta \mid\right\} .
$$

Then $\mathcal{A}_{E}$ is a $\Phi_{E}$-invariant $\mathrm{AF} C^{*}$-subalgebra of $C^{*}(E)$ with $\mathcal{D}_{E} \subset \mathcal{A}_{E}$; hence it follows from (2) that

$$
h_{l}(E) \leq h t\left(\left.\Phi_{E}\right|_{\mathcal{A}_{E}}\right) .
$$


Lemma 3.7. Let $v$ be a vertex of an irreducible graph $E$ with at least two vertices and let $n \geq 1$. Then the elements in the set

$$
\omega(n, v)=\left\{s_{\alpha} s_{\beta}^{*}|r(\alpha)=r(\beta)=v,| \alpha|=| \beta \mid \leq n\right\}
$$

are linearly independent.

Proof. We prove the assertion by induction on $n$. For $n=1$, suppose

$$
x=\sum_{\substack{e, f \in E^{1} \\ r(e)=r(f)=v}} \lambda_{e f} s_{e} s_{f}^{*}+\lambda_{0} p_{v}=0 .
$$

If $e_{0}$ and $f_{0}$ are edges with $r\left(e_{0}\right)=r\left(f_{0}\right)=v$ and either $s\left(e_{0}\right) \neq v$ or $s\left(f_{0}\right) \neq v$, then $s_{e_{0}}^{*} p_{v} s_{f_{0}}=0$; hence

$$
0=s_{e_{0}}^{*} x s_{f_{0}}=\lambda_{e_{0} f_{0}}\left(s_{e_{0}}^{*} s_{e_{0}}\right)\left(s_{f_{0}}^{*} s_{f_{0}}\right)=\lambda_{e_{0} f_{0}} p_{v} ;
$$

thus $\lambda_{e_{0} f_{0}}=0$. Similarly, $\lambda_{e f}=0$ if $e$ and $f$ are loops at $v$ and $e \neq f$. Then $x$ becomes

$$
x=\sum_{e \in E_{l}^{1}(v)} \lambda_{e e} s_{e} s_{e}^{*}+\lambda_{0} p_{v}=0 .
$$

By irreducibility of $E$ and the assumption that $\left|E^{0}\right|>1$, there exists an edge $f$ with $s(f)=v, r(f) \neq v$. Then $s_{f} s_{f}^{*} x=\lambda_{0} s_{f} s_{f}^{*}=0$, so that $\lambda_{0}=0$ and we have $x=\sum_{e \in E_{l}^{1}(v)} \lambda_{e e} s_{e} s_{e}^{*}=0$. Since the projections $\left\{s_{e} s_{e}^{*} \mid e \in E_{l}^{1}(v)\right\}$ are mutually orthogonal, it follows that $\lambda_{e e}=0$ for each $e \in E_{l}^{1}(v)$.

Now suppose that the assertion is true for $n-1$. If

$$
x=\sum_{\substack{|\alpha|=|\beta| \leq n \\ r(\alpha)=r(\beta)=v}} \lambda_{\alpha \beta} s_{\alpha} s_{\beta}^{*}=0, \lambda_{\alpha \beta} \in \mathbb{C},
$$

then for an edge $e \in E^{1}$ we have

$$
0=s_{e}^{*} x s_{e}=\sum_{\substack{\alpha=e \alpha^{\prime} \\ \beta=e \beta^{\prime}}} \lambda_{\alpha \beta} s_{e}^{*} s_{\alpha} s_{\beta}^{*} s_{e}=\sum_{\left|\alpha^{\prime}\right|=\left|\beta^{\prime}\right| \leq n-1} \lambda_{\left(e \alpha^{\prime}\right)\left(e \beta^{\prime}\right)} s_{\alpha^{\prime}}\left(s_{\beta^{\prime}}\right)^{*} .
$$

Note that the elements $s_{\alpha^{\prime}}\left(s_{\beta^{\prime}}\right)^{*}$ appearing in the sum are distinct. Thus by the induction hypothesis, one sees that $\lambda_{\left(e \alpha^{\prime}\right)\left(e \beta^{\prime}\right)}=0$. But the edge $e$ was arbitrary, and so we conclude that the coefficients $\lambda_{\alpha \beta}$ are all zero.

Using the same idea as in the proof of [4, Proposition 2.6] one can prove the following, which is stated in [2] without a proof in the case where $\left\{\omega_{\lambda}\right\}$ is an increasing sequence. We provide a proof only for the reader's convenience.

Proposition 3.8. Let $\Phi: A \rightarrow A$ be a contractive cp map of an exact $C^{*}$-algebra A. If $\left\{\omega_{\lambda}\right\}_{\lambda \in \Lambda}$ is a net (partially ordered by inclusion) of finite subsets in $A$ such that the linear span of $\bigcup_{\lambda, l \in \mathbb{Z}^{+}} \Phi^{l}\left(\omega_{\lambda}\right)$ is dense in $A$, then

$$
h t(\Phi)=\sup _{\lambda} h t\left(\Phi, \omega_{\lambda}\right) .
$$

Proof. Let $\omega=\left\{a_{1}, a_{2}, \ldots, a_{m}\right\}$ be a finite subset in $A$ and $\delta>0$. Then there exists a $\lambda \in \Lambda$ and $p \in \mathbb{N}$ such that if $\bigcup_{0 \leq l \leq p} \Phi^{l}\left(\omega_{\lambda}\right)=\left\{x_{1}, \cdots, x_{k}\right\}$, then

$$
\left\|a_{i}-\sum_{i, j} \lambda_{i j} x_{j}\right\|<\delta
$$


for some $\lambda_{i j} \in \mathbb{C}$. Put $C:=\max _{i, j}\left|\lambda_{i j}\right|$. Choose $(\phi, \psi, B) \in C P A(i d, A)$ with $\operatorname{rank}(B)=\operatorname{rcp}\left(\omega_{\lambda} \cup \cdots \cup \Phi^{p+n}\left(\omega_{\lambda}\right), C^{-1} \delta\right)$. Then for $0 \leq l \leq p+n$,

$$
\begin{aligned}
& \left\|\psi \circ \phi\left(\Phi^{l}\left(a_{i}\right)\right)-\Phi^{l}\left(a_{i}\right)\right\| \\
\leq & \| \psi \circ \phi\left(\Phi^{l}\left(a_{i}\right)-\Phi^{l}\left(\sum \lambda_{i j} x_{j}\right) \|\right. \\
& +\left\|\psi \circ \phi\left(\Phi^{l}\left(\sum \lambda_{i j} x_{j}\right)\right)-\Phi^{l}\left(\sum \lambda_{i j} x_{j}\right)\right\|+\left\|\Phi^{l}\left(\sum \lambda_{i j} x_{j}\right)-\Phi^{l}\left(a_{i}\right)\right\| \\
= & 2 \delta+\left\|\sum_{i, j} \lambda_{i j}\left(\psi \circ \phi\left(\Phi^{l}\left(x_{j}\right)\right)-\Phi^{l}\left(x_{j}\right)\right)\right\| \\
\leq & 2 \delta+\max _{i, j}\left|\lambda_{i j}\right| \cdot C^{-1} \delta=3 \delta .
\end{aligned}
$$

Thus for any $n \in \mathbb{N}$,

$$
r c p\left(\omega \cup \cdots \cup \Phi^{p+n}(\omega), 3 \delta\right) \leq r c p\left(\omega_{\lambda} \cup \cdots \cup \Phi^{p+n}\left(\omega_{\lambda}\right), C^{-1} \delta\right),
$$

which implies that

$$
h t(\Phi, \omega, 3 \delta) \leq h t\left(\Phi, \omega_{\lambda}, C^{-1} \delta\right) .
$$

Therefore we have $h t(\Phi, \omega) \leq h t\left(\Phi, \omega_{\lambda}\right)$.

The AF algebra $\mathcal{A}_{E}$ contains $\Phi_{E}$-invariant AF subalgebras $\mathcal{A}_{E}(v), v \in E^{0}$,

$$
\mathcal{A}_{E}(v):=\overline{\operatorname{span}}\left\{s_{\alpha} s_{\beta}^{*}|r(\alpha)=r(\beta)=v,| \alpha|=| \beta \mid\right\} .
$$

We show that the topological entropy of the restriction map $\left.\Phi_{E}\right|_{\mathcal{A}_{E}(v)}$ has an upper bound $h_{b}\left({ }^{t} E\right)$ which might be strictly smaller than the upper bound for $h t\left(\left.\Phi_{E}\right|_{\mathcal{A}_{E}}\right)$ given in Theorem 3.10.

Proposition 3.9. Let $E$ be an irreducible infinite graph. Then for each $v \in E^{0}$,

$$
h t\left(\left.\Phi_{E}\right|_{\mathcal{A}_{E}(v)}\right) \leq h_{b}\left({ }^{t} E\right) .
$$

Proof. Let $A_{n}(v)$ be the $C^{*}$-subalgebra of $\mathcal{A}_{E}(v)$ generated by $\omega(n, v)$. Then from

$$
s_{\alpha} s_{\beta}^{*} \cdot s_{\mu} s_{\nu}^{*}= \begin{cases}s_{\alpha \mu^{\prime}} s_{\nu}^{*}, & \text { if } \mu=\beta \mu^{\prime}, \\ s_{\alpha} s_{\nu \beta^{\prime}}^{*}, & \text { if } \beta=\mu \beta^{\prime}, \\ 0, & \text { otherwise, }\end{cases}
$$

we see that $A_{n}(v)=\operatorname{span}(\omega(n, v))$ is finite dimensional.

Since $\{\omega(n, v)\}_{n}$ is an increasing sequence of finite subsets in $\mathcal{A}_{E}(v)$ such that the linear span of $\bigcup_{n} \omega(n, v)$ is dense in $\mathcal{A}_{E}(v)$, by Proposition 3.8 it suffices to show that

$$
h t\left(\Phi_{E}, \omega(n, v)\right) \leq h_{b}\left({ }^{t} E\right), \quad n \in \mathbb{N} .
$$

Set $E_{l}^{*}(v):=\bigcup_{k>0} E_{l}^{k}(v)$ and $r(n):=\left|\bigcup_{k=0}^{n} E_{r}^{k}(v)\right|$. Fix $n_{0} \in \mathbb{N}$, and define a map $\phi: \omega\left(n_{0}, v\right) \rightarrow M_{r\left(n_{0}\right)}$ by

$$
\phi\left(s_{\alpha} s_{\beta}^{*}\right)=\sum_{\substack{|\alpha \gamma| \leq n_{0} \\ \gamma \in E_{l}^{*}(v)}} e_{(\alpha \gamma)(\beta \gamma)},
$$

where $\left\{e_{\mu \nu}\right\}$ are the standard matrix units of the matrix algebra $M_{r\left(n_{0}\right)}$. Since the elements in $\omega\left(n_{0}, v\right)$ are linearly independent by Lemma 3.7, one can extend the map to the linear map $\phi: A_{n_{0}}(v) \rightarrow M_{r\left(n_{0}\right)}$. Now we show that $\phi$ is in fact a $*$-isomorphism. To prove that it is a $*$-homomorphism, we only need to see that

$$
\phi\left(\left(s_{\alpha} s_{\beta}^{*}\right)\left(s_{\mu} s_{\nu}^{*}\right)\right)=\phi\left(s_{\alpha} s_{\beta}^{*}\right) \phi\left(s_{\mu} s_{\nu}^{*}\right) .
$$


If $\beta=\mu \beta^{\prime}$, then $s_{\alpha} s_{\beta}^{*} s_{\mu} s_{\nu}^{*}=s_{\alpha}\left(s_{\nu \beta^{\prime}}\right)^{*}$ and

$$
\begin{aligned}
\phi\left(s_{\alpha} s_{\beta}^{*}\right) \phi\left(s_{\mu} s_{\nu}^{*}\right) & =\sum_{\substack{|\alpha \gamma| \leq n_{0} \\
\gamma \in E_{l}^{*}(v)}} e_{(\alpha \gamma)\left(\mu \beta^{\prime} \gamma\right)} \cdot \sum_{\substack{|\mu \delta| \leq n_{0} \\
\delta \in E_{l}^{*}(v)}} e_{(\mu \delta)(\nu \delta)} \\
& =\sum_{\substack{|\alpha \gamma| \leq n_{0} \\
\gamma \in E_{l}^{*}(v)}} e_{(\alpha \gamma)\left(\nu \beta^{\prime} \gamma\right)}=\phi\left(s_{\alpha}\left(s_{\nu \beta^{\prime}}\right)^{*}\right)=\phi\left(s_{\alpha} s_{\beta}^{*} s_{\mu} s_{\nu}^{*}\right) .
\end{aligned}
$$

If $\mu=\beta \mu^{\prime}$, a similar proof works. Otherwise, we have $\phi\left(\left(s_{\alpha} s_{\beta}^{*}\right)\left(s_{\mu} s_{\nu}^{*}\right)\right)=0=$ $\phi\left(s_{\alpha} s_{\beta}^{*}\right) \phi\left(s_{\mu} s_{\nu}^{*}\right)$. In order to show that $\phi$ is injective, let $\phi\left(\sum_{\alpha, \beta} \lambda_{\alpha \beta} s_{\alpha} s_{\beta}^{*}\right)=0$. Then

$$
\sum_{\alpha, \beta} \lambda_{\alpha \beta} \phi\left(s_{\alpha} s_{\beta}^{*}\right)=\sum_{\alpha, \beta} \lambda_{\alpha \beta}\left(\sum_{\substack{|\alpha \gamma| \leq n_{0} \\ \gamma \in E_{l}^{*}(v)}} e_{(\alpha \gamma)(\beta \gamma)}\right)=0 .
$$

But the vectors $\sum_{\substack{|\alpha \gamma| \leq n_{0} \\ \gamma \in E_{l}^{*}(v)}} e_{(\alpha \gamma)(\beta \gamma)}\left(r(\alpha)=r(\beta)=v,|\alpha|=|\beta| \leq n_{0}\right)$ are linearly independent in $M_{r\left(n_{0}\right)}$. In fact, if $A:=\sum_{\alpha, \beta} \lambda_{\alpha \beta}\left(\sum_{\substack{|\alpha \gamma| \leq n_{0} \\ \gamma \in E_{l}^{*}(v)}} e_{(\alpha \gamma)(\beta \gamma)}\right)=0$, then $e_{v v} A e_{v v}=\lambda_{v v} e_{v v}=0$, that is, $\lambda_{v v}=0$, and for any $\alpha, \beta \in E_{r}^{1}(v), e_{\alpha \alpha} A e_{\beta \beta}=$ $\lambda_{\alpha \beta} e_{\alpha \beta}=0$; hence $\lambda_{\alpha \beta}=0$. Repeating the process one has $\lambda_{\alpha \beta}=0$ for any $\alpha, \beta \in \bigcup_{k=0}^{n_{0}} E_{r}^{k}(v)$. Therefore $\sum_{\alpha, \beta} \lambda_{\alpha \beta} s_{\alpha} s_{\beta}^{*}=0$, and the map $\phi$ is injective. The surjectivity of $\phi$ follows from $\operatorname{dim}\left(A_{n_{0}}(v)\right)=r\left(n_{0}\right)^{2}$. We simply write $\phi$ for $\phi: A_{n_{0}+l}(v) \rightarrow M_{r\left(n_{0}+l\right)}(l \geq 0)$, and $\bar{\phi}$ for its contractive cp extension to $\mathcal{A}_{E}(v)$ that exists by Arveson's extension theorem.

For each $n \in \mathbb{N}$ and $0 \leq l \leq n-1$, note that

$$
\bigcup_{l=0}^{n-1} \Phi_{E}^{l}\left(\omega\left(n_{0}, v\right)\right) \subseteq \operatorname{span}\left(\omega\left(n_{0}+n-1, v\right)\right) .
$$

Then the element

$$
\left(\bar{\phi}, \psi:=\phi^{-1}, M_{r\left(n_{0}+n-1\right)}\right) \in C P A\left(i d, \mathcal{A}_{E}(v)\right)
$$

satisfies $\left.\psi \circ \bar{\phi}\right|_{\omega\left(n_{0}+n-1, v\right)}=i d_{\omega\left(n_{0}+n-1, v\right)}$. Thus for each $\delta>0$,

$$
r c p\left(i d, \omega\left(n_{0}+n-1, v\right), \delta\right) \leq r\left(n_{0}+n-1\right),
$$

and so

$$
\begin{aligned}
h t\left(\left.\Phi_{E}\right|_{\mathcal{A}_{E}(v)}, \omega\left(n_{0}, v\right), \delta\right) & \leq \limsup _{n \rightarrow \infty} \frac{1}{n} \log \left(r\left(n_{0}+n-1\right)\right) \\
& =\limsup _{n \rightarrow \infty} \frac{1}{n} \log (r(n)) \\
& =\limsup _{n \rightarrow \infty} \frac{1}{n} \log \left|\bigcup_{k=0}^{n} E_{r}^{k}(v)\right| \\
& =h_{b}\left({ }^{t} E\right) .
\end{aligned}
$$

For the last equality, note that if $k \leq n$, then $\left|E_{r}^{k}(v)\right| \leq\left|E_{r}^{n}(v)\right|$; hence

$$
\left|\bigcup_{k=0}^{n} E_{r}^{k}(v)\right| \leq(n+1) \cdot\left|E_{r}^{n}(v)\right| .
$$


The following theorem gives an upper bound for $h t\left(\left.\Phi_{E}\right|_{\mathcal{A}_{E}}\right)$.

Theorem 3.10. Let $E$ be an irreducible infinite graph and let $\mathcal{A}_{E}$ be the $A F$ subalgebra of $C^{*}(E)$ generated by the partial isometries $\left\{s_{\alpha} s_{\beta}^{*}\left|\alpha, \beta \in E^{*},\right| \alpha|=| \beta \mid\right\}$. Then

$$
h t\left(\left.\Phi_{E}\right|_{\mathcal{A}_{E}}\right) \leq \max \left\{h_{b}\left({ }^{t} E\right), h_{b}(E)\right\}
$$

Proof. Let $E^{0}=\left\{v_{1}, v_{2}, \cdots\right\}$. For each $n_{0} \in \mathbb{N}$ and $n_{1} \in \mathbb{Z}^{+}=\{0\} \cup \mathbb{N}$, put

$$
\begin{aligned}
\omega\left(n_{0}, n_{1}\right) & :=\left\{s_{\alpha} s_{\beta}^{*} \mid \alpha, \beta \in E^{n_{1}}, r(\alpha)=r(\beta) \in\left\{v_{1}, \cdots, v_{n_{0}}\right\}\right\}, \\
\omega_{\Sigma}\left(n_{0}, n_{1}\right) & :=\left\{\sum s_{\alpha_{i}} s_{\beta_{i}}^{*} \mid s_{\alpha_{i}} s_{\beta_{i}}^{*} \in \omega\left(n_{0}, n_{1}\right)\right\} .
\end{aligned}
$$

Note that $\omega_{\Sigma}\left(n_{0}, n_{1}\right)$ is not the linear span of $\omega\left(n_{0}, n_{1}\right)$. Then $\left\{\omega_{\Sigma}\left(n_{0}, n_{1}\right) \mid n_{0} \in\right.$ $\left.\mathbb{N}, n_{1} \in \mathbb{Z}^{+}\right\}$is a net of finite subsets in $\mathcal{A}_{E}$ which is partially ordered by inclusion. In fact, given two finite sets $\omega_{\Sigma}\left(n_{0}, n_{1}\right), \omega_{\Sigma}\left(m_{0}, m_{1}\right)\left(n_{1} \leq m_{1}\right)$, one may write each element $s_{\alpha} s_{\beta}^{*} \in \omega\left(n_{0}, n_{1}\right)$ as

$$
s_{\alpha} s_{\beta}^{*}=s_{\alpha}\left(\sum_{|\mu|=m_{1}-n_{1}} s_{\mu} s_{\mu}^{*}\right) s_{\beta}^{*}=\sum s_{\alpha \mu}\left(s_{\beta \mu}\right)^{*} \in \omega_{\Sigma}\left(m_{2}, m_{1}\right),
$$

where $m_{2}>\max \left\{n_{0}, m_{0}\right\}$ is an integer large enough so that $r(\alpha \mu) \in\left\{v_{1}, \cdots, v_{m_{2}}\right\}$ for any $\alpha \mu$ appearing in the last sum. Then clearly $\omega_{\Sigma}\left(n_{0}, n_{1}\right) \cup \omega_{\Sigma}\left(m_{0}, m_{1}\right)$ is contained in $\omega_{\Sigma}\left(m_{2}, m_{1}\right)$.

Since the linear span of the set $\bigcup_{n_{0}, n_{1}, n} \Phi_{E}^{n}\left(\omega_{\Sigma}\left(n_{0}, n_{1}\right)\right)$ is dense in $\mathcal{A}_{E}$, by Proposition 3.8, we show that for each finite set $\omega_{\Sigma}\left(n_{0}, n_{1}\right)$,

$$
h t\left(\Phi_{E}, \omega_{\Sigma}\left(n_{0}, n_{1}\right)\right) \leq \max \left\{h_{b}\left({ }^{t} E\right), h_{b}(E)\right\} .
$$

If $s_{\alpha} s_{\beta}^{*} \in \omega\left(n_{0}, n_{1}\right), r(\alpha)=r(\beta)=v$, then for $l \leq n-1$,

$$
\Phi_{E}^{l}\left(s_{\alpha} s_{\beta}^{*}\right)=\sum_{|\mu|=l} s_{\mu \alpha} s_{\mu \beta}^{*}=\sum_{|\mu|=l} s_{\mu \alpha}\left(\sum_{\substack{|\nu|=n-l \\ s(\nu)=v}} s_{\nu} s_{\nu}^{*}\right) s_{\mu \beta}^{*}=\sum_{\substack{|\mu \alpha \nu|=n+n_{1} \\|\mu|=l}} s_{\mu \alpha \nu}\left(s_{\mu \beta \nu}\right)^{*}
$$

because $p_{v}=\sum_{\substack{\nu \mid=n-l \\ s(\nu)=v}} s_{\nu} s_{\nu}^{*}$. Hence one sees that

$$
\bigcup_{i=0}^{n-1} \Phi_{E}^{i}\left(\omega_{\Sigma}\left(n_{0}, n_{1}\right)\right) \subseteq\left\{\sum_{|\mu \alpha \nu|=n+n_{1}} s_{\mu \alpha \nu}\left(s_{\mu \beta \nu}\right)^{*} \mid s_{\alpha} s_{\beta}^{*} \in \omega\left(n_{0}, n_{1}\right)\right\} .
$$

Since the set $\left\{s_{\mu} s_{\nu}^{*} \mid \mu, \nu \in \bigcup_{i=1}^{n_{0}} E^{n_{1}+n}\left(v_{i}\right)\right\}$ forms a matrix unit, it generates the $C^{*}$-subalgebra of $\mathcal{A}_{E}$ which is isomorphic to $M_{k_{n}}$, where $k_{n}=\left|\bigcup_{i=1}^{n_{0}} E^{n_{1}+n}\left(v_{i}\right)\right|$. Let

$$
\rho_{n}: \operatorname{span}\left\{s_{\alpha} s_{\beta}^{*} \mid \alpha, \beta \in \bigcup_{i=1}^{n_{0}} E^{n_{1}+n}\left(v_{i}\right)\right\} \rightarrow M_{k_{n}}
$$

be a $*$-isomorphism with the inverse $\rho^{-1}$. Then by Arveson's extension theorem $\rho$ extends to a contractive cp map $\bar{\rho}: \mathcal{A}_{E} \rightarrow M_{k_{n}}$, so that we obtain an element $\left(\bar{\rho}, \rho^{-1}, M_{k_{n}}\right) \in C P A\left(i d, \mathcal{A}_{E}\right)$ such that $\left\|\rho^{-1} \circ \bar{\rho}(x)-x\right\|=0$ if

$$
x \in \bigcup_{i=0}^{n-1} \Phi_{E}^{i}\left(\omega_{\Sigma}\left(n_{0}, n_{1}\right)\right) \subseteq \operatorname{span}\left\{s_{\alpha} s_{\beta}^{*} \mid \alpha, \beta \in \bigcup_{i=1}^{n_{0}} E^{n_{1}+n}\left(v_{i}\right)\right\} .
$$


Hence

$$
\operatorname{rcp}\left(\bigcup_{i=0}^{n-1} \Phi_{E}^{i}\left(\omega_{\Sigma}\left(n_{0}, n_{1}\right)\right), \delta\right) \leq k_{n}
$$

holds for any $\delta>0$. Thus

$$
h t\left(\Phi_{E}, \omega_{\Sigma}\left(n_{0}, n_{1}\right)\right) \leq \limsup _{n \rightarrow \infty} \frac{1}{n} \log \left(k_{n}\right) .
$$

On the other hand, the irreducibility of $E$ implies that there is an $N$ such that $\left|E^{n_{1}+n}\left(v_{i}\right)\right| \leq\left|E^{n_{1}+n+N}\left(v_{1}\right)\right|$ for $1 \leq i \leq n_{0}$. Hence $k_{n}=\left|\bigcup_{i=1}^{n_{0}} E^{n_{1}+n}\left(v_{i}\right)\right| \leq$ $n_{0}\left|E^{n_{1}+n+N}\left(v_{1}\right)\right|$. Therefore

$$
\limsup _{n \rightarrow \infty} \frac{1}{n} \log k_{n} \leq \limsup _{n \rightarrow \infty} \frac{1}{n} \log \left|E^{n}\left(v_{1}\right)\right|,
$$

and the assertion then follows from Proposition 3.5(b).

Example 3.11. Let $E:=E_{\left\{r_{n}\right\},\left\{l_{n}\right\}}$ be Salama's infinite irreducible graph (see [20]). We assume here that $l_{n}+1 \leq l_{n+1}$ for each $n$. There are $r_{k}$ edges from the vertex $k-1$ to $k$, and there is only one path (of length $l_{k}-l_{k-1}$ ) from the vertex $v_{k}$ to $v_{k-1}$.

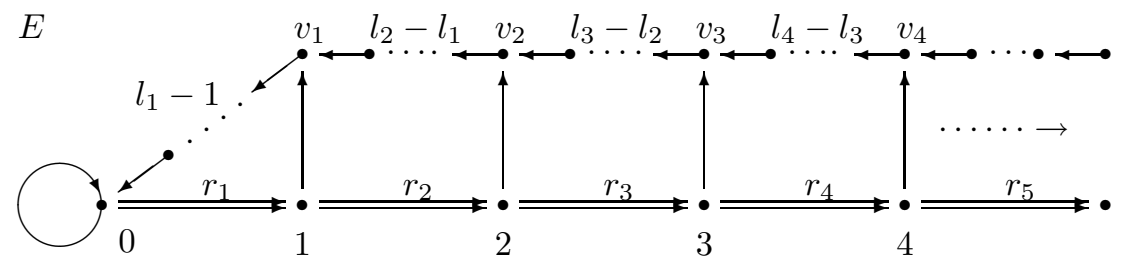

Note that for each $n,\left|E_{r}^{n}(0)^{\star}\right| \leq\left|E_{s}^{n}(0)^{\star}\right|$, which then implies by Proposition 3.2 that

$$
h_{b}\left({ }^{t} E\right) \leq h_{b}(E)
$$

Thus from Theorem 3.10, we have

$$
h t\left(\left.\Phi_{E}\right|_{\mathcal{A}_{E}}\right) \leq h_{b}(E) .
$$

In particular, if $E_{p}:=E_{p, p}(p>1)$ is an irreducible infinite graph of Salama satisfying $h_{l}\left(E_{p}\right)=h_{b}\left(E_{p}\right)=\log p$, by (3) we have

$$
h t\left(\left.\Phi_{E_{p}}\right|_{\mathcal{A}_{E_{p}}}\right)=\log p .
$$

Remark 3.12. After the paper had been submitted, the authors found a meaningful lower bound for $h t\left(\left.\Phi_{E}\right|_{\mathcal{A}_{E}(v)}\right)$ (see Proposition 3.9) and a better upper bound for $h t\left(\left.\Phi_{E}\right|_{\mathcal{A}_{E}}\right)$ in $[12]$.

\section{REFERENCES}

[1] N. P. Brown, K. Dykema and D. Shlyakhtenko, Topological entropy of free product automorphisms, Acta Math. 189 (2002), 1-35. MR.1946916 (2003i:46073)

[2] F. P. Boca and P. Goldstein, Topological entropy for the canonical endomorphism of CuntzKrieger algebras, Bull. London Math. Soc. 32 (2000), 345-352. MR.1747834 (2001f:46097)

[3] T. Bates, D. Pask, I. Raeburn and W. Szymanski, The $C^{*}$-algebras of row-finite graphs, New York J. Math. 6 (2000), 307-324. MR.1777234 (2001k:46084)

[4] N. P. Brown, Topological entropy in exact $C^{*}$-algebras, Math. Ann. 314 (1999), 347-367. MR1697449 (2000g:46093) 
[5] M. Choda, Endomorphisms of shift type (entropy for endomorphisms of Cuntz algebras), Operator Algebras and Quantum Field Theory (Rome, 1996), 469-475, International Press, Cambridge, MA. MR 1491136

[6] J. Cuntz, Simple $C^{*}$-algebras generated by isometries, Comm. Math. Phys. 57 (1977), 173185. MR 0467330 (57:7189)

[7] J. Cuntz and W. Krieger, A class of $C^{*}$-algebras and topological Markov chains, Invent. Math. 56 (1980), 251-268. MR0561974 (82f:46073a)

[8] V. Deaconu, Entropy estimates for some $C^{*}$-endomorphisms, Proc. Amer. Math. Soc. 127 (1999), no. 12, 3653-3658. MR1632272 (2000d:46081)

[9] K. Dykema, Topological entropy of some automorphisms of reduced amalgamated free product $C^{*}$-algebras, Ergodic Theory Dynam. Systems, 21 (2001), 1683-1693. MR.1869065 (2002i:46062)

[10] B. M. Gurevic, Topological entropy of enumerable Markov chains, Dokl. Akad. Nauk SSSR 187 (1969), 216-226. Soviet Math. Dokl. 10 (1969), 911-915. MR0263162 (41:7767)

[11] J. A Jeong and G. H. Park, Topological entropy for the canonical completely positive maps of graph $C^{*}$-algebras, Bull. Austral. Math. Soc. 70 (2004), 101-116. MR2079364 (2005e:46096)

[12] J. A Jeong and G. H. Park, Dynamical systems in graph $C^{*}$-algebras, preprint 2004.

[13] E. Kirchberg, On subalgebras of the CAR-algebra, J. Funct. Anal. 129 (1995), no. 1, 35-63. MR1322641 (95m:46094b)

[14] B. P. Kitchens, Symbolic Dynamics, Springer 1998. MR.1484730 (98k:58079)

[15] A. Kumjian, Notes on $C^{*}$-algebras of graphs, Contemporary Math. 228, Operator Algebras and Operator Theory, 1998, AMS. MR1667662 (99m:46137)

[16] A. Kumjian, D. Pask and I. Raeburn, Cuntz-Krieger algebras of directed graphs, Pacific J. Math. 184 (1998), 161-174. MR1626528 (99i:46049)

[17] A. Kumjian, D. Pask, I. Raeburn, and J. Renault, Graphs, groupoids, and Cuntz-Krieger algebras, J. Funct. Anal. 144 (1997), 505-541. MR.1432596 (98g:46083)

[18] D. Lind and B. Marcus, An introduction to symbolic dynamics and coding, Cambridge University Press 1999. MR.1369092 (97a:58050)

[19] C. Pinzari, Y. Watatani and K. Yonetani, KMS states, entropy and the variational principle in full $C^{*}$-dynamical systems, Commun. Math. Phys. 213 (2000), 331-379. MR1785460 (2002a:46097)

[20] I. A. Salama, Topological entropy and recurrence of countable chains, Pacific J. Math. 134 (1988), 325-341. MR0961239 (90d:54076)

[21] E. Størmer, Entropy of some automorphisms of the $I I_{1}$ factor of the free group in infinite number of generators, Invent. Math. 110 (1992), 63-73. MR1181816 (93i:46112)

[22] D. Voiculescu, Dynamical approximation entropies and topological entropy in operator algebras, Comm. Math. Phys. 170 (1995), 249-281. MR1334396 (97b:46082)

[23] P. Walters, An introduction to ergodic theory, GTM 79, Springer 1982. MR0648108 (84e:28017)

Department of Mathematical Sciences, Seoul National University, Seoul, 151-747 KOREA

E-mail address: jajeong@math.snu.ac.kr

Department of Mathematics, Hanshin University, Osan, 447-791 Korea

E-mail address: ghpark@hanshin.ac.kr 